\title{
EL DISCURSO MÉDICO, PIEDRA ANGULAR DE LA CONSTRUCCIÓN DE LAS RELACIONES DE GÉNERO EN LA ÉPOCA CONTEMPORÁNEA*
}

\author{
Dolores Sánchez \\ Instituto de Estudios de la Mujer de la Universidad de Granada
}

\begin{abstract}
RESUMEN
El concepto de género no establece sólo una distinción entre sexo biológico (dato natural y objeto de conocimiento de la biología y de la medicina) y sexo social (construido dentro de y por relaciones de poder). Como categoría de análisis permite también indagar en el espacio de tensión que gobierna la construcción mutua de uno y de otro. Esa construcción pasa sin lugar a duda por el lenguaje y su capacidad de enraizar en la cultura categorías de pensamiento. Este trabajo pretende poner de manifiesto cómo la categoría lingüística y conceptual de la-mujer es uno de los lugares donde en el siglo XIX se encarna esa construcción mutua.
\end{abstract}

PALABRAS CLAVE: Discurso médico, Siglo XIX, Construcción de las relaciones de género, Las mujeres, La mujer. Análisis del discurso.

\section{MEDICAL DISCOURSE, THE CORNERSTONE OF THE GENDER RELA- TIONS IN CONTEMPORARY TIMES}

\begin{abstract}
The concept of gender establishes not only a distinction between biological sex (natural data and the object of biological and medical knowledge) and social sex (constructed within and by power relations). As a category of analysis, it also allows us to research the area of tension which governs the mutual construction of both. This construction inevitably pervades language and its capacity to establish categories of thought in cultures. This paper will demonstrate how the concep-
\end{abstract}

* Este ensayo se integra en el proyecto de investigación «Medicina y regulación social en España: consensos y resistencias en torno a prácticas científicas sobre las mujeres en los siglos XIX y XX», I+D HUM2006-12278-C03-02 del Ministerio de Educación y Ciencia. Su contenido se deriva del estudio llevado a cabo para la realización de la tesis doctoral, Sánchez, D. (2005) El discurso médico de finales del siglo XIX en España y la construcción del género. Análisis de la construcción discursiva de la categoría la-mujer, Tesis doctoral en CDRom, Granada, Universidad de Granada. 
tual and linguistic category of «Woman» is one of the areas where this mutual construction may be found in the 19th century.

KEY WORDS: Medical discourse, 19th century, Construction of gender relations, Women, Woman, Discourse analysis.

\section{INTRODUCCIÓN}

Desde que las personas nacen, e incluso antes, se establece su identidad mediante las categorías de mujer o varón, obedeciendo a una tradición que, en la cultura occidental, clasifica a las personas en dos entidades sexuales que marcan su devenir social desde su llegada al mundo. En la práctica lingüística de la cultura occidental esta identificación se plasma, en el caso de las mujeres, en un enunciado que pretende significar el conjunto de las mujeres y que en castellano adopta la forma de la estructura lingüística la mujer.

La mujer se entiende, por tanto, como una categoría primaria de catalogación del mundo que nos rodea, es decir, como un dato a priori, de naturaleza ahistórica y que incluye a todas las mujeres. Esa categoría va más allá de la mera referencia a los órganos genitales que, en el mundo occidental, es el rasgo externo corporal, no exento de ambigüedad histórica ${ }^{1}$, sobre el que se hace la distinción entre las personas de un sexo u otro. Como categoría incluye, además, rasgos y características de comportamiento o de tipo psicológico que completan el cuadro que establece las diferencias entre hombres y mujeres. En ese esquema fundamentalmente dual el concepto de mujer está vinculado a una forma de ser que parece determinada por el cuerpo. La continuidad histórica de la expresión lingüística la mujer transporta de un lugar a otro de la historia su carga conceptual y el arsenal de representaciones que alimenta la idea de que la identidad de las mujeres es una identidad anclada al cuerpo. La familiaridad lingüística de la expresión la mujer camufla la consistencia y la densidad con la que la noción de mujer atraviesa la historia, con un carácter aparentemente inmutable, fijo y difícil de desafiar.

Problematizar esa obviedad con la que el conjunto de los discursos sociales vincula esta categoría del lenguaje con una entidad natural del mundo real ha sido tarea de las investigaciones y teorías feministas. Hasta los años setenta las mujeres éramos la mujer. La historia, la sociología, la antropología y muchas otras disciplinas revisadas por el pensamiento feminista han mostrado

1 Fausto-Sterling, A. (2006), Cuerpos sexuados: la política de género y la construcción de la sexualidad, Barcelona, Melusina. 
que la mujer no existía. El feminismo ha construido otro sujeto, el de las mujeres, sujeto histórico, social, político, cultural y, también, estratégico porque necesitaba de una categoría sociológica y política que le permitiera demostrar el carácter social de la exclusión y de la dominación. La categoría las mujeres tampoco es una categoría estable que se dé por sentada. El feminismo es una teoría crítica y, por ello, lleva varios años reflexionando sobre el peligro totalizador de dicha categoría. La crítica feminista posmoderna, en su afán justificado por cercar y desbaratar un repertorio argumentativo esencializador, intenta desplazar la lógica binaria que subyace a categorías básicas como mujer, mujeres o femenino que implican una oposición a hombre, hombres o masculino y plantear marcos de definiciones abiertas que permitan dotar de nuevos significados dichas categorías. Sin embargo, éstas, afincadas históricamente y estructuralmente en las de sexo, siguen siendo aún emblemas potentes de clasificación de lo real y, a pesar de los esfuerzos conjugados por las distintas perspectivas feministas, siguen constituyendo un reducto impregnado de naturalismo. Por eso es necesario seguir desvelando los procesos de construcción histórica de esa naturalización que puedan explicar la fuerza y el arraigo de la lógica de la dominación que se ejerce aún sobre las mujeres como grupo en el marco de las relaciones sociales.

Si la idea de mujer se aferra a nuestra manera de entender el mundo es porque es el resultado de un profundo y complejo proceso de anclaje histórico. En ese proceso el lenguaje, entendido como lugar de constitución y de activación de las categorías con las que nos representamos el mundo, juega un papel determinante. Pero, como todas las categorías del pensamiento, esta es una categoría cargada de historia y en esa historia han participado activamente los discursos científico y médico que, por su estatuto de discursos de la verdad, tienen una gran responsabilidad en el hecho de que se nos imponga, aún en los albores del siglo XXI, la categoría la mujer como categoría ontológica.

Hemos investigado la manera en que el discurso médico construye dicha categoría en la España de finales del siglo XIX a partir de un dispositivo discursivo que tuvo una gran incidencia en su coherencia semántica y que aseguró su anclaje histórico. El objetivo no era hacer un análisis de los contenidos del discurso sino poner al descubierto los mecanismos discursivos que subyacen a esos contenidos y que permiten establecer vínculos unívocos entre lo natural y lo social. A partir de un corpus constituido por textos escritos por médicos españoles, publicados entre el año 1880 y 1895 y que tienen como tema central el estudio de la mujer, hemos analizado la literatura médica producida en un tiempo y espacio determinados, en términos de los intereses políticos que representa y del modelo normativo de identidad que propugna para las mujeres. 


\section{APROXIMACIÓN INTERDISCIPLINAR}

La investigación se sustenta en un marco teórico y metodológico interdisciplinar en el que confluyeron aportaciones de los estudios feministas, de las corrientes de la crítica a la ciencia y del análisis crítico del discurso (ACD). De manera general, estos tres enfoques coinciden en la imbricación de las estructuras sociales, políticas e institucionales en la construcción de la realidad, insistiendo el enfoque del ACD en la dimensión discursiva de esta.

Dadas las derivas múltiples que ha sufrido el concepto de género desde que pasó a ser categoría central de análisis de algunas corrientes del pensamiento feminista, queremos detenernos en aclarar qué entendemos por género. Siguiendo a Joan $\mathrm{Scott}^{2}$ entendemos el género como un elemento constitutivo de las relaciones sociales basadas en las diferencias percibidas entre los sexos y como una forma primaria de relaciones significantes de poder. La definición de Scott es compleja, dialéctica y aboga claramente por un análisis contextualizado:

«Como elemento constitutivo de las relaciones sociales basadas en las diferencias percibidas entre los sexos, el género comprende cuatro elementos interrelacionados: primero, símbolos culturalmente disponibles que evocan representaciones distintas (y a menudo contradictorias) [...] Segundo, conceptos normativos que manifiestan las interpretaciones de los significados de los símbolos, en un intento de limitar y contener sus posibilidades metafóricas. [...] esas declaraciones normativas dependen del rechazo o represión de posibilidades alternativas [...]. Sin embargo, la posición que emerge como predominante es expuesta como la única posible. La historia subsiguiente se escribe como si esas posiciones normativas fueran producto del consenso social más que del conflicto. [...] [El análisis de género] debe incluir nociones políticas y referencias a las instituciones y organizaciones sociales, tercer aspecto de las relaciones de género. [...] El cuarto aspecto del género es la identidad subjetiva. [...] los hombres y las mujeres reales no satisfacen siempre o literalmente los términos de las prescripciones de la sociedad o de nuestras categorías analíticas. [Es necesario] investigar las formas en que se construyen esencialmente las identidades genéricas y relacionar sus hallazgos con una serie de actividades, organizaciones sociales y representaciones culturales históricamente específicas» ${ }^{3}$.

El género es por lo tanto una construcción densa que se mueve y atraviesa todos los niveles de la organización social, cristalizando en una entidad de

2 ScotT, J. W. (1990), «El género: una categoría útil para el análisis histórico». En Amelang, J. y NASH, M. (eds.) Historia y género: las mujeres en la Europa moderna y contemporánea, Valencia, Edicions Alfons El Magnánim, pp. 23-56

3 SCOTT (1990), pp. 45-46. 
aparente estabilidad absoluta en el tiempo y en el espacio. Se entiende así su capacidad de sedimentación a través de la historia y su asentamiento social como estructura mental que marca profundamente nuestra percepción del mundo.

Pero la perspectiva de género invita también a mirar la construcción del género como una construcción dialéctica. El género, por muy denso que sea, contiene fisuras; su marca, por muy profunda, no es indeleble. Las personas, las mujeres, construyen su identidad subjetiva poniendo constantemente en juego la estabilidad del género, a través de la negociación o de la resistencia a normas, comportamientos, discursos que definen masculinidad y feminidad, a lo largo de sus vidas y a lo largo de la historia. La identidad generizada es el resultado de esa contienda que, en función de la eficacia de fuerzas sociales variadas, da al género una apariencia sustancial, controlando lo que es permitido, lo que es deseable, lo que es normal, lo que es femenino...

El concepto de género no establece sólo una distinción entre sexo biológico (objeto de conocimiento de la biología y de la medicina propuesto por ellas como dato natural) y sexo social (construido dentro de y por relaciones de poder). Como categoría de análisis permite también indagar en el espacio de tensión que gobierna la construcción mutua de uno y de otro. Creemos que esta puntualización es necesaria en un momento en el que el género se comprende cada vez más como un hecho positivo que conviene administrar o bien como una serie de identidades cuya producción performativa no tendría más límites que las de nuestra libre elección.

Sobre este planteamiento básico se articulan las otras dos perspectivas disciplinares que completan y consolidan la aproximación al discurso médico en tanto que discurso institucional.

Por una parte la aproximación crítica a la ciencia en la que son particularmente relevantes las aportaciones de la teoría sociológica del llamado constructivismo social ${ }^{4}$. Este enfoque considera la ciencia como una actividad social y cultural que, como tal, no es independiente ni del tiempo, ni del lugar donde se produce, ni de los actores implicados en los procesos de construcción del conocimiento. Con esto se pone bajo sospecha los postulados de objetividad, neutralidad y universalidad que sostienen el andamiaje epistemológico de la ciencia occidental y se indaga en las condiciones que rodean, facilitan o limitan la producción de su discurso. La investigación feminista en este terreno ha puesto de manifiesto, por su parte, cómo la ciencia ha utilizado la polaridad sexual en su

4 Berger, P. y LuCKMAnN, T. ([1966]1994), La construcción social de la realidad, Buenos Aires, Amorrortu editores. 
manera de construir un conocimiento científico marcadamente androcéntrico ${ }^{5}$ y ha cuestionado las nociones mismas de objetividad, racionalidad y universalidad de la ciencia como valores androcéntricos para poner de manifiesto que el conocimiento y la práctica científica han sido un eje mayor de la legitimación de la discriminación sexual en las sociedades occidentales ${ }^{6}$.

La tercera herramienta teórica y metodológica de nuestro estudio está constituida por las diversas aportaciones de los estudios críticos del discurso. Al proponer un concepto de discurso como práctica social imbricada en otras prácticas sociales y que interacciona constantemente con ellas, estos estudios ofrecen herramientas para acercarse de forma fructífera a la relación textocontexto. En este marco se enfoca el corpus discursivo de la investigación (textos médicos sobre «la mujer» del último tercio del siglo XIX) como una secuencia dentro de la producción discursiva global de una sociedad en un momento histórico determinado: un discurso dentro de los discursos. Pero también se entiende como un tipo de discurso caracterizado por y característico de una práctica social particular: la práctica discursiva de la institución médica. Al ser el discurso de una elite con poder social, se pone el énfasis sobre el papel del lenguaje en la producción y reproducción del poder ${ }^{7}$. Por otra parte el ACD permite operar dentro de un marco de análisis que recoge también los elementos perturbadores de la producción y circulación de un discurso particular en el orden social discursivo y revelar los desafíos, las transgresiones, las contradicciones y fisuras que ese orden entraña.

La convergencia teórica de las tres perspectivas utilizadas en la investigación, que se definen asimismo como miradas críticas, permiten, pues, estudiar el papel del discurso médico, en tanto que discurso institucional, en la construcción de la realidad y en concreto de las relaciones de género en tanto que estas son también, y junto con otros niveles de análisis, una construcción discursiva. Pero quiero volver a subrayar que la apuesta por la utilización de las herramientas teóricas y metodológicas del ACD respondía también a la necesidad de conseguir articular poder del discurso y resistencia, es decir indagar en el espacio de tensión entre, por una parte, los discursos normativos y sus propuestas sobre el género $\mathrm{y}$, por otra, las disidencias sociodiscursivas que integran y alimentan de forma paradójica su hegemonía.

5 Keller, E. F. (1991), Reflexiones sobre género y ciencia, Valencia, Alfons El Magnànim.

6 Harding, S. (1996), Ciencia y feminismo, Madrid, Morata.

7 VAN DiJK, T. A. (2000a), «El discurso como interacción en la sociedad». En VAN DiJK, T. A. (coord.) El discurso como interacción social. Estudios sobre el discurso II, Barcelona, Gedisa, pp. 19-66. 
Por lo tanto es este marco interdisciplinar en el que los acercamientos son complementarios y confluentes, lo que me ha permitido acercarme a esa densidad y a esa comprensión dialéctica de la construcción de las relaciones de género a las que remitía más arriba.

\section{LAS HIPÓTESIS DE TRABAJO}

Los trabajos realizados en historia de la medicina sobre discurso médico sobre las mujeres en el siglo XIX y cambio del XIX al XX ${ }^{8}$, así como la consulta sistematizada de fuentes bibliográficas ${ }^{9}$ y posterior búsqueda y localización de materiales me llevaron a formular mis hipótesis de manera más precisa.

Existe una polarización del discurso médico sobre lo que el propio discurso denomina como la mujer. Si bien esa concentración de interés es constante a lo largo del siglo XIX, se incrementa significativamente en los treinta últimos años del siglo. Esta inflación discursiva podría ser muy significativa desde el punto de vista de su análisis como fenómeno discursivo-social en el marco de una sociedad en plena transformación económica y social.

La coincidencia entre esta polarización y la incorporación del positivismo a la medicina española en el último tercio del siglo pudo afectar las prácticas discursivas de la comunidad médica dando lugar a procedimientos lingüístico-discursivos diferenciados o propios del discurso científico en relación con el desarrollo de una retórica de la verdad.

Por último y partiendo de la idea de que la relación entre sexo y género no es del orden de lo natural sino una elaboración socio-lógica (es decir que se inserta en una lógica social), la existencia de un discurso médico específico sobre lo que el discurso mismo denomina la mujer daba lugar a pensar que esta noción encarnada en el lenguaje fuera un lugar importante donde esta correlación axiomática entre sexo y género se articulara.

Esto nos llevó a seleccionar un conjunto de textos médicos producidos entre 1880 y 1895 y cuyo valor principal reside en su representatividad como discurso que afirma su voluntad de hablar «con conocimiento» de la mujer. El

8 Para una revisión historiográfica reciente de esas fuentes ver Ortiz Gómez, T. (2006), Medicina, historia y género. 130 años de investigación feminista. Oviedo, Ediciones KRK, pp170-177.

9 El Manual del Librero Hispanoamericano de Antonio Palau y Dulcet, Empuries: Palau Dulcet, 1986 y el Index-Catalogue of the Library of the Surgeon. Nueva-York: Johnson reprint Corporation, 1972 [1910]. 
corpus estaba compuesto de diversos géneros textuales: artículos publicados por sus autores en prensa profesional, monografías, capítulos de libro, conferencias publicadas en actas de congreso, publicaciones escritas de discursos orales pronunciados por sus autores en las sesiones de apertura dos grandes instituciones médicas de la época - la Sociedad Ginecológica Española y la Real Academia de Medicina de Madrid. En algunos casos, un mismo texto había sido publicado varias veces y en soportes textuales distintos. Esto, que constituye una muestra de la inflación discursiva a la que nos referíamos más arriba, fue un elemento importante, significativo en cuanto al alcance de la difusión del discurso, que se tomó en cuenta en el análisis ${ }^{10}$.

Nos acercamos a la resolución de estas hipótesis utilizando los textos producidos no como fuentes para conocer la argumentación científica desplegada por el discurso médico sobre las mujeres sino para problematizar la trama y la textura discursiva misma. Es pertinente, como señala van Dijk ${ }^{11}$, subrayar la importancia para los historiadores de recordar la naturaleza textual de las fuentes. Es decir, cuestionar su condición de intérpretes y testimonios privilegiados y fiables de su época y poner de manifiesto su carácter instrumental en la producción y reproducción del poder en tanto que lugares de negociación de significados sociales importantes.

Siguiendo las propuestas del ACD, la metodología seguida intenta poner de manifiesto la interacción constante entre texto y contexto, tanto al nivel de lo macrodiscursivo, esto es el funcionamiento del discurso médico estudiado en el conjunto de los discursos sociales, como a nivel microdiscursivo, esto es la textura interna del discurso en torno a la categoría de la mujer. Por lo tanto la utilización del contexto no tiene como finalidad pintar un decorado que serviría de telón de fondo al análisis discursivo sino sacar a escena las propiedades de la situación social que fueron relevantes para entender la existencia de un discurso médico sobre las mujeres, las condiciones que determinaron su producción y distribución social así como su configuración interna. Siguiendo a van Dijk ${ }^{12}$, hacer operativa la confrontación entre situación contextual y discurso es condición sine qua non para entender el discurso como interacción, es decir como el resultado de acontecimientos sociales y/o discursivos que incidieron en él, cuestionándolo o consolidándolo. Es, igualmente, condi-

10 Para una descripción más detallada del corpus de fuentes analizadas ver SÁNCHEZ (2005), pp. 92-118.

11 VAN DiJK, T. A. (2000b), «El estudio del discurso». En VAN DiJK, T. A. (coord.) El discurso como estructura y proceso. Estudios sobre el discurso I, Barcelona, Gedisa, p. 56.

12 VAN DiJK (2000a). 
ción para entenderlo como acción, es decir con capacidad para tener efectos en lo social.

\section{EL CONTEXTO}

Expondremos muy brevemente dos aspectos contextuales esenciales para el análisis. Por una parte, la cuestión de las mujeres porque son el objeto del discurso y, por otra, la situación, en ese momento, de la institución médica por ser esta la productora del discurso estudiado.

En resumen, se puede decir que después de treinta años de investigación en Historia de las mujeres es insostenible la idea de que las mujeres del siglo XIX se subsumían en la figura de ángel del hogar transportada hasta nuestros días por la historiografía tradicional ${ }^{13}$. El colectivo de las mujeres fue una pieza clave del ordenamiento económico y social de la época. La industrialización del país desplazó a las ciudades grandes sectores de población rural para formar la mano de obra reclamada por la industria. Un número considerable de mujeres se incorporó al trabajo de la fábrica y a las profesiones que se desarrollaron en el marco del proceso paralelo de urbanización. En ese contexto, las mujeres de las clases acomodadas accedieron a algunos ámbitos profesionales reservados hasta el momento a los hombres. Esas mismas mujeres alzaban su voz para reclamar, a través de las numerosas tribunas en las que participaron, el derecho a la educación y al ejercicio profesional. Es significativa la coincidencia en el tiempo entre el discurso sobre la mujer producido por la institución médica y la existencia de varios textos de mujeres de la época que cuestionaban la función social de lo que ellas también denominaban «la mujer» ${ }^{14}$. Las mujeres, por tanto, estaban en todas partes, sujetos de la

13 La puesta en escena de las mujeres como agentes sociales fundamentales en la España de finales del siglo XIX, implicadas en prácticas sociales alejadas de lo que el conocimiento histórico y enciclopédico tradicional nos ofrece como visión de la época, no hubiera sido posible sin la ingente labor historiográfica feminista que invalida la representación historiográfica dominante en la que las mujeres están ausentes. Para una visión general de esa cuestión en el siglo XIX ver Duby, G. y Perrot, M. (dirs.) (1991), Historia de las mujeres en Occidente. Vol.4, Madrid, Taurus y Morant, I. (dir.) (2006) Historia de las mujeres en España y América Latina. Vol. III Del siglo XIX a los umbrales del XX. Madrid, Cátedra.

14 Entre otros destacan los de Concepción Arenal, Emilia Pardo Bazán y Berta Wilhelmi de Dávila: Arenal, C. ([1868]1993), «La mujer del porvenir. Comentario de Isabel Cabrera». En DuRÁn, M. A. (coord.), Mujeres y hombre. La formación del pensamiento igualitario, Madrid, Castalia, 73-82; CABRERA BOSCH, M. I. (1988). «Las mujeres que lucharon solas: 
historia con prácticas sociales muy diversas y, también, sujetos de discurso, de un discurso que cuestionaba el status quo de la llamada condición femenina. Todo esto contrasta con el lugar que les reservaba el proyecto social que los grupos dominantes estaban diseñando. En este proyecto se pretendía que las mujeres asumieran gratuitamente el trabajo doméstico y la reproducción de la familia.

La institución médica, por su parte, era un espacio de prestigio y autoridad social en pleno crecimiento. En su proceso de expansión y asentamiento, la medicina se fue dotando de una estructura científica cada vez más fuerte que separó saber académico y saber popular, limitó el acceso al conocimiento científico a través de la institucionalización de los estudios médicos y controló el ejercicio de las profesiones vinculadas a la salud ${ }^{15}$. El discurso médico era, por lo tanto, un discurso bajo control. El vínculo estrecho entre la comunidad médica y los grupos sociales con poder económico garantizaba su promoción y distribución. Era, finalmente, un discurso de gran trascendencia social: la medicina no sólo se constituyó en torno a un cuerpo de conocimiento que legitimaba su posición social y determinaba el poder de su discurso sino que, también, era una práctica. La interacción constante entre profesionales y usuarios lo convertía en un poderoso instrumento de difusión ideológica. Además, su compromiso afirmado con el progreso y bienestar de la humanidad justificaba su intromisión en las estructuras sociales. Estaba, pues, en una posición privilegiada desde la cual disparar toda una batería de propuestas normativas en cuanto al género, es decir en cuanto al papel social que debían desempeñar las mujeres por ser mujer ${ }^{16}$. Esto lo convirtió en piedra angular

Concepción Arenal y Emilia Pardo Bazán». En FolguerA, P. (comp.), El feminismo en España: dos siglos de historia, Madrid, Editorial Pablo Iglesias, 29-50; SCANLON, G. M. (1989). «Ideología y experiencia femenina en la tribuna de Emilia Pardo Bazán». En MAQUIEIRA D'ANGELO, V. (ed.), Mujeres y hombres en la formación del pensamiento occidental. Actas de las VII Jornadas de investigación interdisciplinaria, Madrid, Universidad Autónoma de Madrid, 289-299; WiLHelmi DE DÁvila, B. ([1893]1993). «Razón del movimiento en favor de la mujer. Comentario de Teresa González Calbet». En DuRÁN, M. A. (coord.), 83-98.

15 Sobre esta cuestión, BYNuM, W. (1994), Science and the Practice of Medicine in the Nineteenth Century, Cambridge, Cambridge University Press.

16 Entre otros trabajos, ver los de BORDERIES-GuERENA, J. (1989), «El discurso higiénico como conformador de la mentalidad femenina (1865-1915)». En MAQUIEIRA D'ANGELO, V. (ed.), Mujeres y hombres en la formación del pensamiento occidental. Actas de las VII Jornadas de investigación interdisciplinaria, Madrid, Universidad Autónoma de Madrid, pp. 309299; RuIZ SomaviLLA, M J J. (1994), «La legitimación de la ideología a través de la ciencia: la salud y la enfermedad de la mujer en el Siglo Médico». En De la Ilustración al romanticismo. 
de un edificio discursivo sobre el que se asentaron otros discursos sociales. El contexto histórico, social e institucional que explica el protagonismo de la medicina así como su hegemonía social en aquella época determina el impacto del discurso que se produce en su seno. Determina, por consiguiente, la eficacia social con la que pudo imponer y afincar sus propuestas normativas en cuanto a la configuración de una identidad generizada de las mujeres.

\section{HEGEMONÍA Y CONTIENDA DISCURSIVA}

El análisis macrodiscursivo llevado a cabo sobre el corpus aspira a salir de la inmanencia de los significados textuales, buscando en las condiciones históricas de la enunciación los elementos que determinan su coherencia y su hegemonía, al tiempo que imprimen su marca sobre la textura discursiva. El corpus discursivo estudiado cobra significación a partir de su inserción en el contexto que he prefigurado anteriormente. Su aprehensión en la red global de su intertextualidad social es lo que permite ver cómo emerge como región discursiva específica en colaboración con, frente a, o contra otros discursos sociales. Por otra parte, y en una dimensión histórica, el discurso, como señala Fairclough ${ }^{17}$ es el resultado de otros discursos anteriores y punto de partida de discursos futuros o posibles. Este acercamiento incluye, por tanto, las disidencias sociales y los discursos que estas provocan. El discurso médico sobre la mujer se asienta como hegemónico a través del dispositivo que estructura el orden social discursivo. Un orden que absorbe, bloquea, modifica o da paso de manera reglada a los conceptos diseminados en el conjunto de los discursos sociales ${ }^{18}$.

Si bien la capacidad del discurso de construir una representación de la $m u$ jer se asienta en la posición de fuerza social de los enunciadores del discurso, esta construcción, sin embargo no es mecánica y pasa por una gestión sociodiscursiva de lo que se enuncia. El dispositivo que se organiza tiende al acaparamiento legitimado de la cuestión de la mujer por la comunidad médica. A las estrategias de legitimación/deslegitimación, autorización/desautorización

Cádiz, América y Europa ante la Modernidad: VII Encuentro La mujer en los siglos XVIII y XIX, Cádiz, Universidad de Cádiz, pp. 103-114 y Ruiz Somavilla, Mª J. y JiMÉNEZ LuCENA, I. (1994), «La construcción de la patología femenina en la España contemporánea». En RAmos Palomo, D. (coord.), Femenino plural. Palabra y memoria de mujeres, Málaga, Universidad de Málaga, pp. 235-250.

17 FAirclough, N. (1989), Language and Power, Londres, Longman, p.154.

18 Martín Rojo, L. (1997), «El orden social de los discursos». Discurso (México), 21/22, pp. 13-30. 
discursiva, y silenciamiento de discursos disidentes, en particular el discurso de las propias mujeres, se añaden estrategias de fabricación del consenso social en pro del equilibrio social y del progreso humano. Este primer movimiento tiende a configurar un orden discursivo en el que la comunidad médica se apropia y monopoliza la capacidad de hablar sobre la mujer.

El análisis demuestra que la hegemonía del discurso no se establece mecánicamente y es el resultado de una contienda social y discursiva. El contexto, y particularmente el hecho de que las mujeres fueran protagonistas activas de su tiempo, provoca el discurso. Lo provoca porque convoca su existencia y lo provoca porque lo hostiga. Para ilustrar esto, tomaremos un ejemplo que confronta el texto de la maestra y libre pensadora granadina Bertha Whilelmi publicado en 1893 y que se llama "Aptitud de la mujer para todas las profesiones» ${ }^{19}$ y seis de los artículos que integran nuestro corpus de análisis, publicados entre 1894 y 1895, esto es, después del de Wilhelmi, en una de las revistas profesionales más importantes del momento, El Siglo Médico, por tres autores distintos y que, al presentarse su publicación a modo de debate, tienen todos el mismo título «¿Puede ejercer la mujer las diversas profesiones del hombre?»" ${ }^{20}$.

Brevemente se puede decir que frente al texto de Wilhelmi, que es anterior a los seis textos médicos, que es asertivo y no sesgado cuando afirma la capacidad de las mujeres para ejercer todas las profesiones, los textos de nuestros médicos pretenden invalidar la aserción proclamada por Wilhelmi pasando a una retórica interrogativa que pone en duda esa capacidad de las mujeres afirmada por la autora. Ellos, los médicos, tienen el poder institucional y epistemológico para plantear cuáles son las preguntas: la interrogación se sobrescribe y, así, emborrona la aserción de Wilhelmi.

Esta confrontación entre el texto de Wilhelmi y los seis textos médicos es sólo una muestra de que no hay consenso social; lo que hay es un ruido discursivo más fuerte que pretende controlar una insurrección social, que es también una insurrección discursiva, la de las mujeres.

Pero este control del flujo discursivo es sólo el primer paso. En el contexto de crisis ocasionado por la presencia insubordinada de las mujeres en la socie-

19 Wilhelmi De Davila, B. ([1893]1993), pp. 83-98.

20 En orden cronológico de publicación: Rojo PRIETO, C. (1894), «¿Puede ejercer la mujer las diversas profesiones del hombre?». El Siglo Médico, 41, 462-463; HERnÁNDEZ SANTOS, S. (1894), «Idem». El Siglo Médico, 41, 574-575; SÁnCHEZ-COMENDADOR, A. de. (1894), «Idem». El Siglo Médico, 41, 607; Rojo Prieto, C. (1895), «Idem». El Siglo Médico, 42, 270-271; Hernández Santos, S. (1895), «Idem». El Siglo Médico, 42, 509-510 y SáNCHEZ-COMENDADOR, A. de. (1895), «Idem». El Siglo Médico, 42, 302-203. 
dad de finales del siglo XIX, el discurso médico necesitaba de un dispositivo que diera firmeza a afirmaciones apodícticas sobre la mujer y asegurara su estabilidad epistémica. Ese proceso, complementando los anteriores, se asienta en reglas discursivas de segregación internas al discurso y llega a su apogeo con la apropiación misma del objeto del discurso, la mujer, que es convertido en categoría propia y exclusiva del escrutinio científico. La posición de dominio que emerge de la oposición entre discurso médico y otros discursos sociales se asienta en la irrefutabilidad de los enunciados porque estos se derivan de un modelo de conocimiento que se da como el único capaz de dar cuenta de la verdad de los secretos de la naturaleza. A continuación un ejemplo que ilustra un momento de este proceso en un texto de Álvarez Espino:

\begin{abstract}
«(...) el alma de la mujer entró en los dominios de la ciencia. Desde entonces su suerte estaba asegurada. (...) La mujer había caído en poder del sabio, su existencia había logrado ser pensamiento del filósofo, su destino había adquirido la consistencia de un problema antropológico y su sentimentalismo, su inteligencia, su conciencia, su alma entera, debía ser objeto de observación y análisis; se había de registrar y de escudriñar, latido por latido, aliento por aliento, misterio por misterio, y, ya contadas sus pulsaciones, descubiertas sus tendencias, apreciadas sus aptitudes, revelado su arcano, la mujer entraba en la vida racional, se alzaba a la altura del hombre, en los dominios al menos de la especulación, conseguía su puesto en la ciencia, en la economía, en la política, y compartía con su redentor filosófico el imperio de ese gran centro en la esfera de los intereses sociales, del movimiento espiritual y del destino humano. La mujer estaba salvada» ${ }^{21}$.
\end{abstract}

En el modelo de conocimiento positivista, el científico se convierte en un simple intérprete de lo que dice la naturaleza. Sólo se trataría, como se aprecia en la cita que sigue de un texto de del Bustos, de poner palabras a lo que la naturaleza misma dispone con empeño ante el ojo observador:

«(...) para que la diferencia en lo anatómico entre el hombre y la mujer sea aun más patente, existe todavía otra mucosa que lo revela (...). La trama de los tejidos de la mujer es menos apretada, como demostrando menos aptitud para la fuerza» ${ }^{22}$.

21 ÁlVAREZ Espino, R. (1880), «Insuficiencia de la educación actual de la mujer, exponiendo la que debe de ser en el porvenir». En Actas de las sesiones del Congreso Regional de Ciencias Médicas celebrado en Cádiz en los días 10, 11, 12, 13 y 14 de agosto de 1879, Cádiz, Casa Editorial de D.F. de P. Jordan, pp. 163-175, p. 166.

22 Del Busto, A. (1892), «Problemas morales, sociales y políticos que resuelve el estudio médico de la mujer». En Discursos leídos en la sesión inaugural del año de 1892 de la Real Academia de Medicina, Madrid, Establ. tipo. Enrique Teodoro, p. 32. 
Desde el punto de vista del funcionamiento del discurso, se puede decir que la herramienta textual ha acumulado ya una serie de datos transhistorizados en torno a la categoría del lenguaje la mujer pero su puesta en funcionamiento dentro de un discurso específico - el discurso médico sobre la mujer - la construye con un valor pragmático añadido al constituirlo como categoría científica ${ }^{23}$. La categoría la-mujer ${ }^{24}$ se convierte en un elemento central del funcionamiento del discurso en tanto que categoría taxonómica de lo real.

La relación entre la entidad extralingüística las mujeres y el referente del discurso ${ }^{25}$, la-mujer, no traduce a nuestro entender un simple desliz semántico, e identifico aquí el nudo gordiano con el que el discurso médico liga y sujeta el sexo al género y viceversa. Nuestro análisis pretende ser operativo a partir de esa disyunción clara entre entidad y referente lingüístico del discurso. La entidad es ese sujeto/objeto definido histórica y sociológicamente como el actor social las mujeres ${ }^{26}$ en la sociedad española de finales del siglo XIX y el referente es la marca lingüística que codifica esa entidad.

La función social e ideológica del dispositivo macrodiscursivo es la de construir una representación de las mujeres que tenga capacidad normativa y pueda crear identidades conformes con las necesidades de los grupos sociales dominantes de la época. Como dice Martín Rojo ${ }^{27}$, el discurso tiene poder porque los modos de objetivación pasan a ser modos de subjetivación. Las estrategias discursivas que configuran la representación apelan a la identificación de los sujetos con las proposiciones contenidas en el discurso. Aunque este propósito del discurso en general y del discurso médico estudiado se realiza implícitamente - y de ahí su eficacia - la siguiente cita de un texto del médico Álvarez Espino, publicado en 1880, es su expresión explicita:

23 Sobre este procedimiento ver Gross, A. G. (1990), The Rhetoric of Science, Cambridge (Massachussets), Harvard University Press, p. 44.

24 A partir de ahí tomamos la decisión de representar gráficamente esta operación de anclaje lingüístico mediante la grafía la-mujer que es también un intento por nuestra parte de hacer más evidente la naturalización del concepto a través de su forma lingüística.

25 Sobre la cuestión de la relación entre referente y entidad ver VAN DIJK (2000b), p. 33 y sobre la problematización de los significados asignados, FAIRCLOUGH (1989), pp. 94-95.

26 Esto no presupone que le atribuyamos a la categoría de las mujeres un sentido estable. Si bien el feminismo ha recurrido a esa categorización como base de fortalecimiento de sus propias exigencias políticas de visibilidad y de transformación, se está generando en la actualidad un inmenso debate sobre lo que constituye o debería constituir la categoría de las mujeres. Ver, por ejemplo, BUTLER J. (2001), El género en disputa, Barcelona, Paidós, 33-37.

27 MARTín ROJO (1997), p. 29 
«Preciso es que la mujer tenga una idea exacta de sí y disponga de medios eficaces de defensa contra el mundo. La idea de sí sólo puede dársela la ciencia. (...) Hagámosla ante todo moral, para que sea buena: pero no entendamos que es más buena ni más moral la que más reza, sino la que tiene mejor y más clara conciencia de su deber» ${ }^{28}$.

\section{COHESIÓN Y FISURAS DEL DISCURSO}

La resolución ideológica completa de la categoría la-mujer pasa por su cristalización en la configuración semántica que emana de la textura discursiva. Para analizar su fuerza ideológica en tanto que categoría normativa nos centraremos en la manera en la que es codificada por el discurso mediante el análisis de las marcas léxicas utilizadas para denominar a las mujeres y de su relación isotópica ${ }^{29}$ con la categoría central la-mujer. Es decir, observamos cómo el colectivo de las mujeres es referido por el discurso a partir del estudio del despliegue referencial y focal ${ }^{30}$ en torno a lo que Ruth Wodak denomina «topos de definición» o lugar común donde se articula la definición del grupo y que parafraseando a la autora se puede hacer explícito con la siguiente regla: si un grupo de personas (las mujeres) es nombrado/designado como la-mujer, ese grupo de personas es portador de las cualidades/rasgos/atributos contenidos en el sentido (literal) de la-mujer ${ }^{31}$.

A partir de la localización de las denominaciones distintas que nombran a las mujeres, del recuento de sus ocurrencias y de la observación de las estrategias que vinculan estas denominaciones entre ellas se puede trazar el mapa de la representación de las mujeres por el discurso. Ésta pasa por una primera operación que es central y sobre la que se superponen los demás procedimientos. Esta operación es la utilización preferente, masiva y reiterativa del singular «la mujer» ${ }^{32}$.

\footnotetext{
28 Álvarez EsPino (1880), p. 173.

29 Sobre el estudio de las relaciones isotópicas en el discurso, CALSAMiglía BLANCAFORT, H. y Tusón VAlls, A. (1999), Las cosas del decir. Manual de análisis del discurso, Barcelona, Ariel, pp. 230-236.

30 Ver Tomlin, R. S. et al. (2000), «Semántica del discurso». En VAN DiJK (2000a), pp. 107-170.

31 WODAK, R. (2001), «The discourse-historical approach». En WodAK (ed.) Methods of Critical Discourse Analysis, Londres, Sage, pp. 63-94, p. 75.

32 En el artículo 'Muger' del Diccionario de ciencias médicas publicado en 1824, es decir unos 60 años antes del corpus analizado, se aprecia una distribución mucho menos estandarizada del singular frente al plural mucho más frecuente en el texto de cuarenta páginas que constituye dicho artículo.
} 
A partir de ahí diversos procedimientos, entre los cuales la sinonimia, la metonimia, la hiponimia o la utilización del singular, con los que se manejan las denominaciones de referencia a las mujeres dibujan una representación heterosocial de las relaciones de género sobre la base de una carta de naturaleza de lamujer que integra fundamentalmente los significados de madre y esposa. Esto asegura el colapso entre sexo y género, naturalizando la función social de la maternidad y vinculándola al destino biológico. El número muy reducido de otras denominaciones genéricas como «la mitad de la humanidad», «el sexo» bello o débil, o «hembra» confirma la categoría de «la mujer» como pieza central en la construcción de la representación de las mujeres. Las denominaciones que remiten a relaciones sociales de las mujeres limitan estas al ámbito del parentesco o de la familia. Están prácticamente excluidas de la representación las mujeres de las clases trabajadoras. Son una ausencia, un hueco de la representación. A través de procedimientos como la modalización, la utilización del plural, la asociación con lo extranjero o lo monstruoso, la descarga y recarga semántica, ese mismo mapa tiende a excluir en un movimiento centrífugo de la representación todo lo que no remite a esa carta de naturaleza que etiqueta lo que es o debe de ser la-mujer: las mujeres como grupo social, las mujeres que ejercían o pretendían ejercer una profesión, las mujeres concretas y reales (personalidades de la época), las solteras, las extranjeras que son un mal ejemplo...

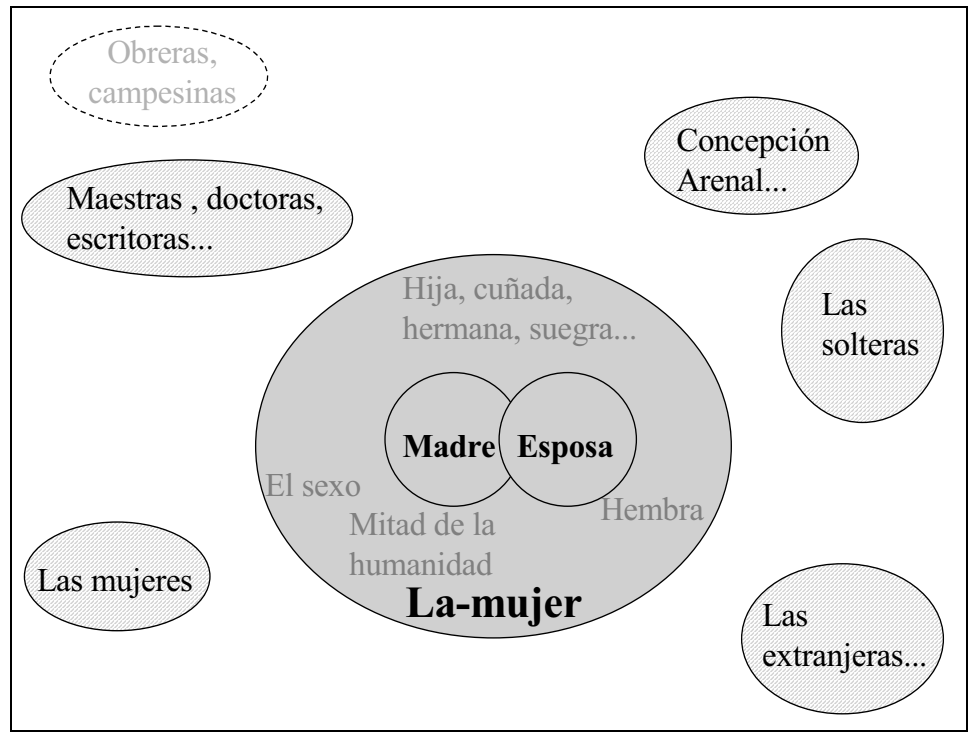

Carta de naturaleza de la-mujer 
De ese mapa, la representación que emerge es la de la mujer como entidad abstracta, monolítica, homogénea, dependiente de los hombres, ahistórica, asocial, acultural y sin agencia social e histórica. La confrontación textocontexto demuestra, sin embargo, que lejos de ser una construcción aleatoria e inocente las elecciones rutinarias y trivializadas del discurso en el modo de referenciar a las mujeres responden a una voluntad de crear, es decir de construir, algo que no existe naturalmente.

En la cita siguiente ejemplifico una estrategia de relación metonímica entre la categoría principal de referencia, la mujer, y otro referente en el discurso, la madre:

«La mujer, y hablo en tesis general, no tiene aptitud para los trabajos intelectuales que requiere el provechoso cultivo de las ciencias, según resulta de su estudio anatómico, fisiológico y psico-fisiológico; pero esto debe importar muy poco, le debe tener muy tranquila, porque si bien es cierto que al genio corresponden lauros y honores, respeto, amor y gloria son en alto grado patrimonio de la madre $)^{33}$.

Esa rutina metonímica es constante y satura el discurso estudiado. Tiene como efecto el de elaborar la naturalización de las mujeres no sólo como reproductoras de la especie sino también como criadoras. Esto es, como madres biológicas y como madres sociales. En el contexto de la España de finales del siglo XIX, esa relación carecía de obviedad ${ }^{34}$ y por ello el discurso se esfuerza en construirla. Este es un ejemplo entre otros muchos, de una estrategia entre otras muchas. Es el conjunto de estas estrategias que acumuladas y reiteradas dan su carácter y su fuerza performativa a la representación, es decir su capacidad de construir la realidad/verdad.

Lo que el análisis microdiscursivo demuestra también es que a pesar del poder del discurso para excluir o distorsionar la representación de las mujeres reales, este no puede contener la realidad de la presencia activa de las mujeres en la sociedad española de finales del siglo XIX. El ejemplo siguiente es de un texto del médico Rojo Prieto publicado en prensa médica:

33 RodRíguez Rubí y PACHECO, Á. (1882), «Educación e instrucción a que debe someterse la mujer para cumplir sus altos sinos sociales». Discurso doctrinal por ---. Discursos leídos en la sesión inaugural de la Sociedad Ginecológica Española el día 8 de diciembre de 1882. Madrid, Gómez de Fuentenebro, p. 60.

34 Para un interesante debate ver Bock, G. y Thane, P. (eds.) (1996) Maternidad y politicas de género: la mujer en los estados de bienestar europeos, 1880-1950, Madrid, Cátedra. 
«Si lo pretendido por la mujer fuese justo [ejercer una profesión], podríamos pasar por alto; pero es injusto a todas luces, y nosotros vamos a probar su imposibilidad, sin temer las iras que podríamos despertar, no sólo en las Emilias, Rosarios, y otras señoras, sino en esa pléyade de marimachos que se llaman yankeesas» ${ }^{35}$.

Desde el punto de vista de la situación comunicativa en la que está implicado este enunciado, es decir un artículo publicado por su autor médico en la prensa profesional, es decir $s u$ prensa, en la que se dirige a sus colegas, el tono, o más bien la salida de tono, el nerviosismo de este enunciado desmiente la estabilidad de la posición superior e intocable del enunciador y de su discurso. En este sentido he podido afirmar más arriba que las prácticas sociales y discursivas de las mujeres provocan y exacerban el discurso. La elección de los dos nombres propios, Emilias y Rosarios, que están en cursiva en el original, no es casual. Es claramente una alusión contextual a todas aquellas mujeres ${ }^{36}$ que como Emilia Pardo Bazán o Rosario de Acuña defendieron con tesón e inconformismo la emancipación de las mujeres y amenazaron el orden social establecido.

\section{CONCLUSIÓN}

A pesar de la carga de obviedad significativa que pueda haber acumulado el enunciado la mujer para todos y todas nosotras se puede desentrañar su construcción histórica generizada en la entretela del discurso médico de finales del siglo XIX en España. Se puede decir que la explosión del discurso médico sobre la mujer a finales del siglo XIX fue ocasionada por la presencia insubordinada de las mujeres en la sociedad decimonónica española. El discurso emergente sobre la mujer fue el resultado de una intersección sociodiscursiva conflictiva. Su producción y configuración dependieron tanto del poder hegemónico de la institución médica como de las mujeres que lo confrontaron y lo resistieron. El triunfo discursivo de la-mujer como enunciado lingüístico condensó la naturalización de la posición social de las mujeres. Al amarrar solidamente el colapso entre sexo biológico y sexo social, la-mujer se convirtió en una categoría útil para la construcción patriarcal de las relaciones de género y por ello constituye una de las grandes herramientas de la lógica

35 Rojo PRIETO, (1894), p. 462.

36 Ver MARTíneZ, C. et al. . (dirs.). (2000), Mujeres en la Historia de España. Enciclopedia biográfica, Barcelona, Planeta. 
de la dominación patriarcal. Sin embargo desentrañar su construcción generizada - es decir insertada en la lógica de unas relaciones asimétricas y de poder entre los sexos - muestra que no había ninguna coacción natural que forzara a las mujeres a asumir un determinado modelo de identidad. El análisis crítico del discurso, aplicado aquí en una dimensión histórica, muestra que la pervivencia de la categoría la mujer que aún atraviesa las ciencias contemporáneas es una manifestación del poder del discurso. Su fuerza reside precisamente en su capacidad de convencernos de que no es discurso. La resistencia de muchas ciencias a aceptar la novedad epistemológica del género no es más que una manifestación del poder del discurso para negar su carácter constructivo.

Fecha de recepción: 19 de abril de 2007

Fecha de aceptación: 15 de junio de 2007 